\title{
The Curling Probe: a numerical and experimental study. Application to the electron density measurements in an ECR plasma thruster
}

\author{
Federico Bonif Julien Jarrige, and Victor Desangles \\ DPHY, ONERA, Université Paris-Saclay, F-91123 Palaiseau, France \\ Tiberiu Minea \\ Laboratoire de Physique des Gaz et des Plasmas, CNRS, Université Paris 91405, Orsay, France
}

(Dated: December 10, 2020)

\begin{abstract}
The measurement of electron density is a key issue in understanding and controlling plasma applications. To date, plasma density in electric thrusters has been mainly evaluated with electrostatic techniques, such as the Langmuir probe, which could be quite invasive. In this paper, we propose the application of a microwave resonant probe, the curling probe, to the diagnostic of an electrodeless plasma thruster. The measurable electron density range and the probe accuracy are found to be limited by the probe natural frequency. We present the numerical study and the experimental characterization of three curling probes with different natural frequencies (700, 1400 and $3000 \mathrm{MHz}$, approximately). First, an analytical equation of the natural frequency as a function of geometrical parameters is drawn from 2D numerical simulations. Then, a procedure based on solid dielectric etalons is proposed for the absolute calibration of the probe. Finally, measurements are performed in the plume of an ECR plasma thruster. Electron densities from $10^{8}$ to $10^{11} \mathrm{~cm}^{-3}$ have been obtained, in agreement with hairpin and Langmuir probes results. A wall-embedded probe has enabled measurements inside the thruster source with minimal plasma perturbation. A possible curling probe configuration, embedded in a reactor wall, is proposed as a fully non-invasive diagnostic for plasma sources.
\end{abstract}

\section{NOMENCLATURE}

\section{$\alpha, \beta \quad 3 \mathrm{D}$ geometric factors}

$\Delta f \quad$ frequency shift, $\mathrm{Hz}$

$\sigma, \sigma_{(.)} /($.$) absolute, relative uncertainty$

$\tan \delta \quad$ electric loss tangent

$\varepsilon_{0} \quad$ permittivity of free space, $\mathrm{F} / \mathrm{m}$

$\varepsilon_{d} \quad$ real part of the relative permittivity

$\varepsilon_{p} \quad$ plasma permittivity

$\varepsilon_{r} \quad$ relative permittivity

$B \quad 2$ D geometric factor

$d \quad$ aperture diameter, $\mathrm{m}$

$d_{\text {ant }} \quad$ monopole antenna diameter, $\mathrm{m}$

e electron charge, $\mathrm{C}$

$f^{\lambda / 4}, f^{\lambda / 2}$ quarter-, half-wavelength resonant frequency, $\mathrm{Hz}$

$f_{0} \quad$ vacuum reference resonant frequency, $\mathrm{Hz}$

$f_{m} \quad$ measured frequency, $\mathrm{Hz}$

$f_{p} \quad$ plasma frequency, $\mathrm{Hz}$

$f_{R} \quad$ resonant frequency, $\mathrm{Hz}$

$$
\begin{array}{ll}
K & \text { probe sensitivity, } \mathrm{Hz} / \mathrm{m}^{-3} \\
L & \text { resonator length, } \mathrm{m} \\
m_{e} & \text { electron mass, } \mathrm{kg} \\
n_{e} & \text { electron density, } \mathrm{m}^{-3} \\
P_{a b s} & \text { absorbed microwave power, } \mathrm{W} \\
T_{a}, T_{p} & \text { ambient, probe temperature, }{ }^{\circ} \mathrm{C} \\
w & \text { slot-antenna width, } \mathrm{m}
\end{array}
$$

\section{INTRODUCTION}

The diagnostics of plasma parameters, such as the electron density and temperature, is a key issue in understanding the plasma features and controlling it for application optimizations, e.g. plasma-aided materials processing, electric propulsion plasma thrusters, etc. Available electron density diagnostic tools suited for electric plasma thrusters include electrostatic probes, such as the Langmuir probe, spectroscopy techniques, and microwave resonant probes.

Langmuir probe (LP) is the simplest and most commonly used method to measure local values of electron density and electron temperature in plasmas. Several models have been validated for the analysis of experimental data, and there is an extensive literature on LP use in various types of plasmas [1]. Although appropriate for spatially-resolved measurements, Langmuir probes can be quite intrusive due to the presence of a voltage-biased metallic tip in the plasma and to the collection of a current up to a few mA. Plasma perturbation and probe damage may become problematic in regions of high particle density and temperature, or if the probe is exposed 
to particles beams, either energetic electrons or ions. Chen et al. [2] pointed out that LP electron density measurements are subject to uncertainty because the several theories commonly used to interpret data may give a wide range of different results. They compared several LP analysis theories with microwave interferometry [3] and plasma-oscillation probes [4], and concluded that Langmuir original OML (Orbital Motion Limited) theory works best if the probe is made as thin as possible.

Non-invasive diagnostics based on spectroscopy or interferometry techniques are also available. Optical emission spectroscopy involves the analysis of spectral lines of excited species. The diagnostic is simple to implement, but it is lineof-sight based, and it relies on complex collisional-radiative models that requires that all spectroscopic constants and cross sections are known [5, 6]. Interferometry technique use the phase shift of a radiation through a plasma to determine electron density. This line-of-sight technique can be very accurate and uses a simple analysis method, but the plasma volume must be large enough and uniform, and the spatial resolution is limited by the frequency range of the interferometry wave. Thomson scattering [7] is largely used for fusion devices diagnostics and allows the determination of the electron density and temperature. It is non-invasive and allows for spatiallyresolved measurements. Though, it is a complex system owing to the need of an optical bench composed of multiple elements and it may require long acquisition time for densities under $10^{10} \mathrm{~cm}^{-3}$ due to low signal intensities. Typical measured values [8, 9] are between $5 \times 10^{10} \mathrm{~cm}^{-3}$ to $10^{15} \mathrm{~cm}^{-3}$ for the density and a few eV for electron temperatures. Recently, Vincent et al. [10] designed a new Thomson scattering technique giving access to electron densities down to $10^{10} \mathrm{~cm}^{-3}$.

In 1976, Stenzel [11] proposed a microwave resonator probe, later called hairpin (HP): a quarter-wavelength openended transmission line in the form of a U-shaped antenna inductively excited by a loop. Microwave probes are characterized by a resonant frequency that depends on the dielectric constant of the surrounding medium. The resonant frequency shifts in proportion to the plasma electron density. This technique allows spatially resolved density measurements in weakly magnetized, low-temperature plasma. The hairpin has been since revisited, with dc-biased version proposed in an unpublished work [12], and different coupling modes and operating modes [13]. The length of the antenna arm, which depends on the working frequency, limits the spatial resolution and can disturb the measurements [14].

In 2011, Liang et al. [15] proposed a new type of microwave resonator probe: a spiral-shaped slot-type antenna, also called the curling probe $(\mathrm{CP})$, for measurements in reactive plasmas. The resonator is excited by a monopole antenna in its central aperture. The planar geometry allows the probe to be directly embedded in a reactor wall, for instance, as opposed to the hairpin probe. The probe behavior was simulated in presence of plasma using 3D Finite Difference Time Domain (FDTD) computing. Two types of resonances were distinguished: the high frequency resonance (HRF), which is produced by the quarter-wavelength standing wave inside the resonator slot and occurs at densities up to $10^{11} \mathrm{~cm}^{-3}$, and the low frequency resonance (LRF), which is a surface wave resonance inside the aperture region that happens even for densities larger than $10^{11} \mathrm{~cm}^{-3}$. Arshadi and Brinkmann [16] studied the unfolded 2D curling probe by means of 2D FDTD simulations and mode matching technique. They demonstrated that LRF frequencies match with the surface wave equation [17]. Later, they [18] completed this analysis for the HRF in a simulated Argon-discharge reactive plasma. The frequency shift increased as the pressure decreased. They concluded that the $\mathrm{CP}$ is well suited for low-pressure plasma: at higher pressures, the plasma becomes a lossy medium due to the strong increase in the collision frequency, resulting in an exponential decay of the EM field inside the probe.

Recently, the curling probe has been applied to the characterization of reactive plasmas for industrial processing [14, [19. 21], where LP measurements were found to be difficult to use [19]. Time-resolved measurements of the electron density have been performed in a pulsed DC discharge in various gases under surface magnetic confinement [14]. Densities as low as $10^{9} \mathrm{~cm}^{-3}$ have been measured with a $100 \mathrm{~mm}$-long and $16 \mathrm{~mm}$ in diameter $\mathrm{CP}$ coated with Alumina $\left(\mathrm{Al}_{2} \mathrm{O}_{3}\right)$. Most recently, the group of Chubu University in Japan performed CP measurements to monitor the dielectric layer deposited during plasma processing in a $13.56 \mathrm{MHz} \mathrm{RF}$ inductive plasma discharge. Hotta et al. [20] used a CP in the chamber wall of the reactor and measured a frequency shift of $230 \mathrm{MHz}$, corresponding to a density of approximately $2.9 \times 10^{10} \mathrm{~cm}^{-3}$ using the CP method proposed by Liang et al. [15]. Ogawa et al. [21] developed a technique to measure the thickness of the deposited film and the electron density simultaneously using two different-sized CPs. The measured density was in the order of $10^{9} \mathrm{~cm}^{-3}$, in agreement with the typical values encountered in their conditions.

Application to electric propulsion. The measurement of the electron density for plasma thrusters and more generally for electric thrusters has mainly been performed using Langmuir probes [22]. Lobbia and Beal [23] give an insight of the recommended practices when applying LP technique to electric propulsion testing. Microwave techniques may also be employed: interferometry, allowing non-invasive measurements [24], and resonant probes, such as the hairpin. Electric thruster performances are quite sensitive to the local perturbation induced by a diagnostic probe: current collection and secondary electron emission from a bare metallic surface. Moreover, when dealing with low-powered thrusters, a good spatial resolution is required.

The objective of this work is to improve the physical understanding of the curling probe and to assess its performances for the characterization of a plasma thruster. First, 2D numerical simulations are used to analyze the resonant behavior of the spiral slot as a function of geometrical parameters. Then, more complex 3D simulations are performed with the full probe structure. These simulations are used to propose an absolute calibration method. In section III we present an experimental validation of the calibration, and an analysis of the probe performances as a function of working frequency, including sensitivity and measurement uncertainty. Finally, in section IV the curling probe is applied to the measurement of 
electron density in an ECR plasma thruster, both in the plume and in the plasma source.

\section{THE CURLING PROBE: PRINCIPLE AND NUMERICAL STUDY}

Active plasma resonance spectroscopy includes microwave resonant probes techniques. They rely upon the creation of a stationary volume wave in-between resonator ends and are available in under-dense plasma, i.e. $f>f_{p}, f_{p}$ being the plasma frequency (Equation 1, and $f$ the microwave frequency.

$$
f_{p}=\frac{1}{2 \pi} \sqrt{\frac{e^{2} n_{e}}{m_{e} \varepsilon_{0}}}
$$

where $m_{e}, e$ and $\varepsilon_{0}$ are the electron mass and charge and the permittivity of free space, respectively. Plasma properties, such as the electron density, can be evaluated from the resonant frequency. In the case of a hairpin probe, the resonator is a parallel-wire quarter-wavelength U-shaped antenna, defined by a length $L$ and a wire spacing $w$. This resonator is usually excited by an inductively coupled loop, and the electric field is maximum at the open end and zero at the short end, yielding a resonant frequency at $f^{\lambda / 4}=c / 4 L$. Another common case of resonator is the half-wavelength resonance between two shorted ends: the maximum electric field is then located at the center of the resonator, while the field is null at the closed ends, yielding a resonant frequency at $f^{\lambda / 2}=c / 2 L$. In the case of a HP immersed in a uniform medium of relative permittivity $\varepsilon_{r}$, volume waves yield a dipole resonance at:

$$
f_{R}=\frac{c}{4 L \sqrt{\varepsilon_{r}}}
$$

In a cold plasma with permittivity $\varepsilon_{r}=\varepsilon_{p}$, the resonance frequency is increased from the vacuum reference value $f_{0}=\frac{c}{4 L}$ to $f_{R}^{2}=f_{0}^{2}+f_{p}^{2}$, hence, plasma density writes:

$$
n_{e}=\frac{4 \pi^{2} m_{e} \varepsilon_{0}}{e^{2}}\left(f_{R}^{2}-f_{0}^{2}\right)
$$

We remind that Equation 3 stands for a cold, uniform and low magnetized plasma.

The behavior of a curling probe is less straightforward: it presents an exciting capacitive coupled aperture connected to a shorted-end spiral; therefore coupling effects may appear due to the spiral geometry. One open question when designing a curling probe is the value of the vacuum reference resonant frequency, which appears to be a key parameter for the upper and lower plasma density limits that can be measured. In this work, we propose a numerical study performed via Finite Element Method based electromagnetic simulation (FEM-em) to investigate the nature of the $\mathrm{CP}$ resonator. The selected approach is that of increasing complexity: starting from a $2 \mathrm{D}$ model to investigate the nature of the resonator, and then focusing on the full simulation of the probe in $3 \mathrm{D}$.
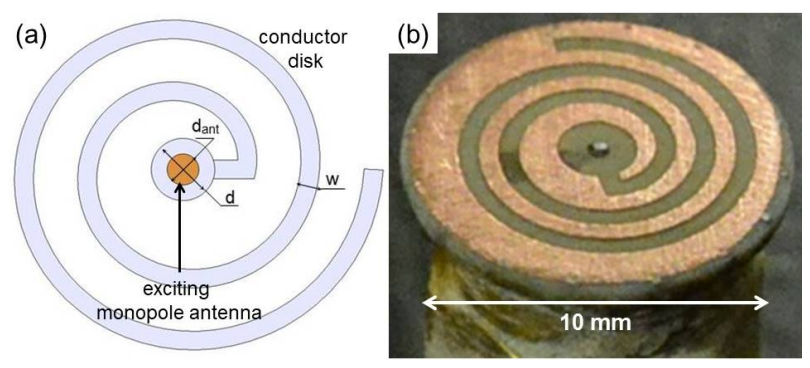

FIG. 1. (a) Schematics of 2D spiral resonator capacitively coupled with the exciting aperture; (b) manufactured CP1400.

\section{A. Two-dimensional analysis}

The simple 2D configuration is here studied: an exciting aperture with a monopole antenna added to a 2D spire (Figure 1(a)). FEM based eigenfrequency analysis have been performed for several geometries of the curling probe in order to determine the nature of the resonator. Simulations have been run on a data pool ranging from $10 \mathrm{~mm}$ to $25 \mathrm{~mm}$ for the resonator length $(L)$ for three aperture diameters $d(1,2$, and $3 \mathrm{~mm})$, for different width-to-aperture ratios $(w / d)$ and antenna-to-aperture ratios $\left(d_{\text {ant }} / d\right)$.

Nature of the resonator. Results, shown in Figure 2, indicate that the quarter or half-wavelength nature mainly depends on the width-to-aperture ratio $(w / d)$, for a given resonator length $L$. Figure 3 gives an insight of the formation of the standing wave inside the spiral slot: the normalized electric field along the center line of the spiral resonator slot is shown, for three different width-to-aperture ratios $(x / L=-1$ and $x / L=1$ correspond to the closed end and the exciting aperture of the spiral, respectively). When the width-to-aperture ratio $(w / d)$ is small, the spiral behaves like a quarter-wavelength resonator: the "open end" is connected to a closed domain several times larger than the resonator width. On the other hand, when the width-to-aperture ratio $(w / d)$ is large, the exciting aperture domain is comparable in dimension with the resonator width and a half-wavelength standing wave occurs. For an intermediate case the waveform would be in-between the half and the quarter-wavelength resonance.

Analytical expression of the resonance frequency in the case of a $2 D$ resonator. An analytical equation (Equation 4 ) for the $2 \mathrm{D}$ resonant frequency is derived from simulation data as a function of three geometrical parameters $w / d, d_{\text {ant }} / d$, $d / L$.

$$
\begin{aligned}
f_{R}^{2 D} & =f^{\lambda / 2}-\frac{f^{\lambda / 4}}{1+\frac{w / d}{B}} \\
B & =2 \frac{d}{L}\left(1-\left(\frac{d_{\text {ant }}}{d}\right)^{2}\right)
\end{aligned}
$$

Equation 4 has been tested outside the original database with satisfying results (prediction error $\leq 3 \%$ ), with resonator lengths $(L)$ ranging from $5 \mathrm{~mm}$ to $50 \mathrm{~mm}$ and aperture diameter $(d)$ from $0.5 \mathrm{~mm}$ to $5 \mathrm{~mm}$, yielding frequencies up to $18 \mathrm{GHz}$. Furthermore, no pattern emerges when the error 
(a)

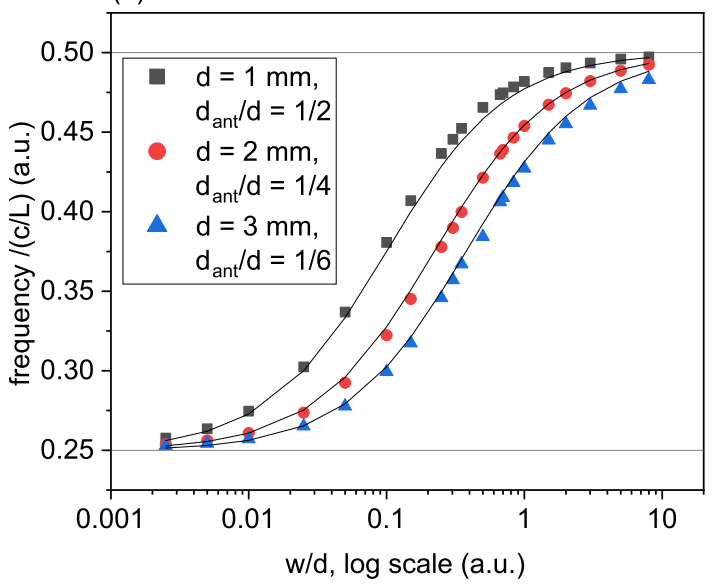

(b)

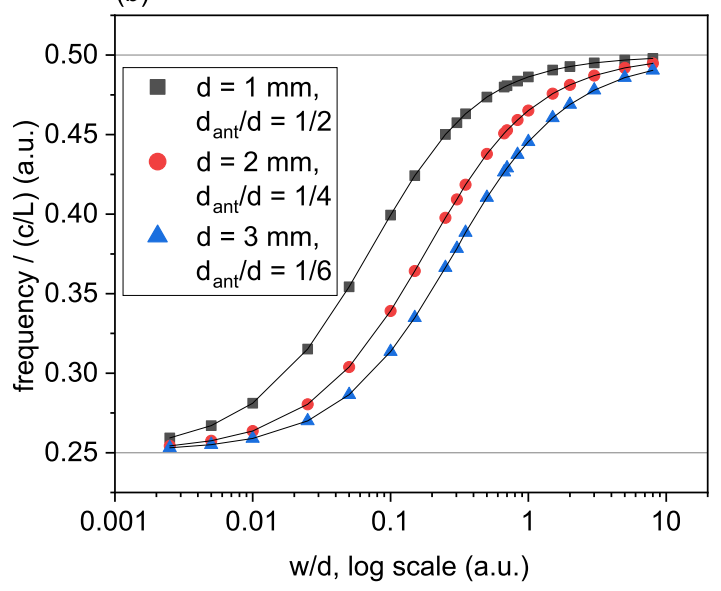

FIG. 2. Non-dimensional frequency for a resonator length of $L=10$ $\mathrm{mm}$ (a) and $L=20 \mathrm{~mm}$ (b). Scatter points: numerical simulation, solid lines: model of Equation 4

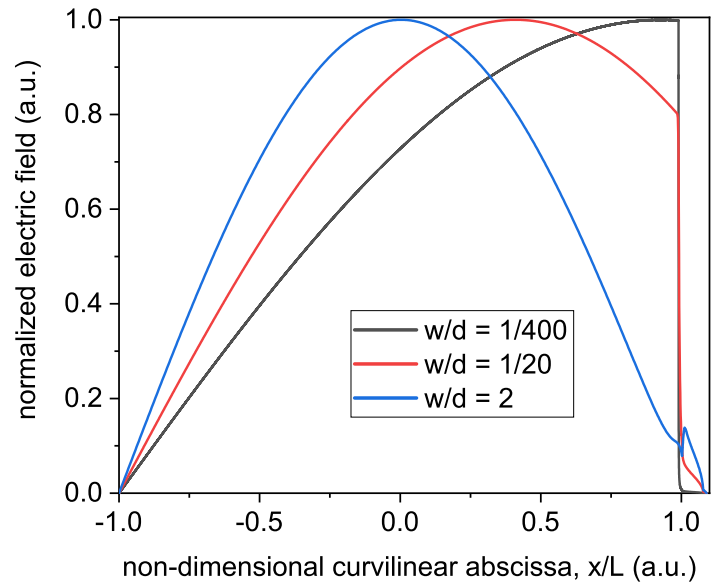

FIG. 3. Effect of the width-to-aperture ratio $(w / d)$, for a given resonator length $L$ and antenna diameter $d_{a n t}$ on the normalized electric field along the curvilinear abscissa. associated to the prediction of Equation 4 is plotted against the three different ratios diameter-to-length $(d / L)$, width-tolength $(w / L)$ and antenna-to-width $\left(d_{\text {ant }} / w\right)$, which may be physically important for the quarter or half wavelength nature.

These conclusions are consistent with previous works on the CP. Using 3D FDTD simulations Liang et al. [15] concluded that the aperture works as an "open end", i.e. the resonator is a quarter-wavelength type. The authors considered a curling probe having a slot width $w=300 \mu \mathrm{m}$ and aperture diameter $d$ varying from $2.4 \mathrm{~mm}$ to $3.6 \mathrm{~mm}$, corresponding to a width-to-aperture ratio $(w / d)$ of 0.125 and 0.083 , respectively. This set of parameters yields a quarter-wavelength nature. Moreover, they also found that when the aperture diameter is increased, the frequency decreases. On the other hand, Arshadi et al. [25] showed that the aperture acts as a "short end" for practical probe dimensions ( $L=35$ to $50 \mathrm{~mm}$, $d<5 \mathrm{~mm}$ and $w<500 \mu \mathrm{m})$, therefore concluding that the curling probe acts as a half-wavelength resonator. Their parametric study (Figure 5 in [25]) shows that for a given length $L$, as the aperture diameter approaches zero, the resonant frequency tends to the half-wavelength resonant value, while as it increases, the resonant frequency tends towards the quarterwavelength value. Besides, this effect is enhanced when the slot width $(w)$ is increased from $50 \mu \mathrm{m}$ to $500 \mu \mathrm{m}$.

\section{B. Three-dimensional analysis}

Proceeding in the numerical analysis, the 3D effects of the probe are here discussed. Figure 4 shows the CAD model of the curling probe and the cut plane visualization of the computed electric field with the tetrahedral mesh used by the solver. 3D numerical simulations are performed with frequency sweep studies: the solver evaluates the power reflection coefficient at the feeding coaxial port over a discrete range of frequencies. The minimum of the reflectance spectrum identifies the resonant frequency of the probe.

The resonance frequency in the $3 D$ case. The vacuum reference frequency in the $3 \mathrm{D}$ case differs from the $2 \mathrm{D}$ value of Equation 4 The 3D resonant frequency is a few hundreds $\mathrm{MHz}$ lower, depending on substrate thickness, substrate permittivity and cavity height. Figure 5 shows how the aperture (at $x / L=1$ ) perturbs the electric field inside the 3D resonator slot. The normalized E-field in the $2 \mathrm{D}$ case is plotted in dashed line for an immediate comparison. It is worth mentioning that the dependency to the 2D-parameters, $w / d$, $d_{\text {ant }} / d$ and $d / L$, is unaltered. The analytical relationship between the 3D and the 2D case is currently under investigation: cavity height, probe-to-antenna diameter ratio and substrate thickness seem to have a major role.

Design of the curling probes. As previously mentioned, the measurable electron density range is strongly affected by the vacuum reference frequency. For this purpose, in this work, we propose to study three different curling probes with different working frequencies (approximately $700 \mathrm{MHz}, 1400$ $\mathrm{MHz}$, and $3000 \mathrm{MHz}$ ), yielding three different behaviors in presence of plasma. In this paper, we refer to the three probes as CP700, CP1400, and CP2965, having a resonator length of 
TABLE I. Geometry of the CP700, CP1400 and CP2965 used for the 3D numerical simulations.

\begin{tabular}{cc}
\hline \hline parameter & value \\
\hline substrate thickness & $0.5 \mathrm{~mm}$ \\
substrate permittivity & 4.5 \\
cavity height & $6 \mathrm{~mm}$ \\
aperture diameter $d$ & $2 \mathrm{~mm}(1 \mathrm{~mm}$ for the CP700) \\
antenna-to-aperture ratio $d_{\text {ant }} / d$ & 0.5 \\
\hline \hline
\end{tabular}

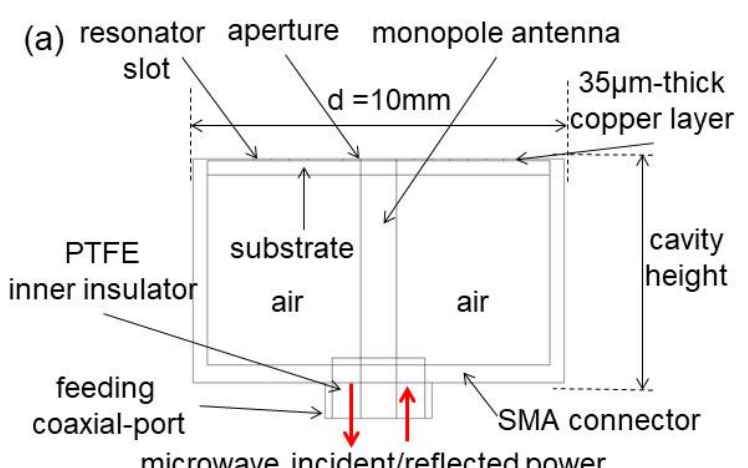

microwave incident/reflected power

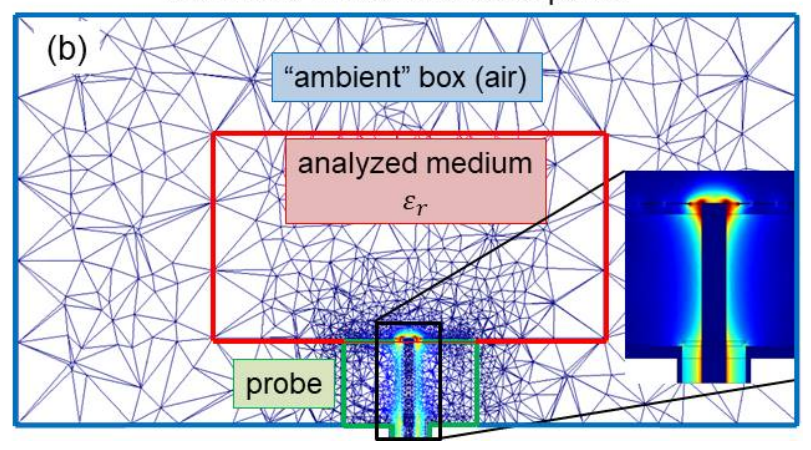

FIG. 4. (a) The CAD model used for 3D numerical simulation of the CP1400; (b) the cut plane visualization of the electric field resulting from the FEM-em simulation at $1000 \mathrm{MHz}$ of the CP1400.

106,44 , and $18 \mathrm{~mm}$, respectively. The geometry used for 3D numerical simulations is fixed as showed in Figure 4 (a) and summarized in Table I.

Calibration procedure. The response of the probes when exposed to a medium (analyzed medium in Figure 4(b)) was simulated for different cases:

- lossy-dielectric materials (and air), with permittivity $\varepsilon_{r}=\varepsilon_{d}(1-j \tan \delta)$, where $\tan \delta$ is the loss tangent accounting for the dielectric losses;

- homogeneous, collisionless, and unmagnetized cold plasma, with permittivity $\varepsilon_{r}=\varepsilon_{p}=1-\left(\frac{f_{p}}{f}\right)^{2}$.

Typical simulated spectra are shown in Figure 6 (a). As expected, the resonant frequency is shifted towards higher frequencies (with respect to air) in presence of plasma, and towards lower values in presence of a solid lossy-dielectric medium.

As in the well-known operational mode of the HP, the CP can be immersed in the plasma medium to be operated. In the

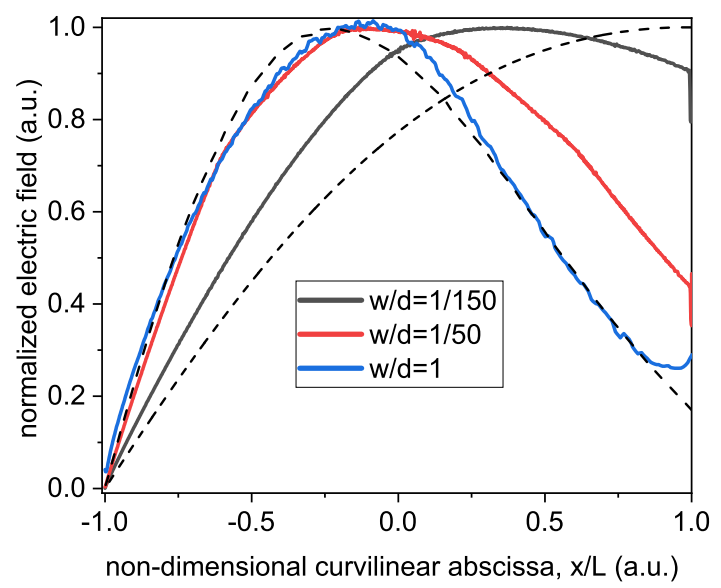

FIG. 5. Effect of the width-to-aperture ratio $(w / d)$ on the electric field in the CP1400 probe. Solid lines: 3D simulations. Dashed line: $2 \mathrm{D}$ case for $w / d=1,1 / 150$.

case of a curling probe, one of the main differences with respect to the hairpin probe is that only one face of the probe is exposed to the plasma, so that the resonant frequency not only depends on the permittivity of the analyzed medium, but also on the permittivity (and geometry) of the structure underneath the resonator slot, partly because of 3D coupling effects between the spiral branches. The analytical relationship between the $3 \mathrm{D}$ and the $2 \mathrm{D}$ resonant frequency (Equation 4 ) being still unknown, we proceeded with the following path. Equation 4 is rewritten in the form:

$$
f_{R}=\frac{c}{2 L \beta \sqrt{\frac{\varepsilon_{r}+\alpha}{2}}}
$$

where $\beta \propto\left(1-\frac{1 / 2}{1+\frac{w / d}{B}}\right)^{-1}$ contains the $2 \mathrm{D}$ geometric factor appearing in Equation 4 , and the effective dielectric constant $\varepsilon_{e f f}=\left(\varepsilon_{r}+\alpha\right) / 2$ accounts for both the dielectric medium upright the probe $\left(\varepsilon_{r}\right)$ and the dielectric substrate structure underneath the resonator slot $(\alpha)$. The geometrical dependency (to probe diameter and aperture diameter $d$ ) of the resonant frequency is consistent to what is found in previous works [15].

First, we simulated probe response for different analyzed medium: vacuum, PTFE, PMMA, Boron Nitride (BN) and MACOR. These materials have been chosen to have different dielectric and loss properties; the $\alpha$ and $\beta$ factors are therefore obtained by fitting procedure following Equation 6 over the computed dielectric data points $\left(f_{R}, \varepsilon_{d}\right)$.

$$
\varepsilon_{r}=(1+\alpha)\left(\frac{f_{0}}{f_{R}}\right)^{2}-\alpha
$$

where $f_{0}=c /(2 L \beta \sqrt{(1+\alpha) / 2})$ is the vacuum reference frequency. Equation 6 is the explicit form of Equation 5 giving the relative permittivity of the medium analyzed by the probe.

Figure 6 presents the results of 3D simulation of CP1400 with solid dielectrics and plasma. It can be seen that the probe response model (Equation 6 has a very good precision for 

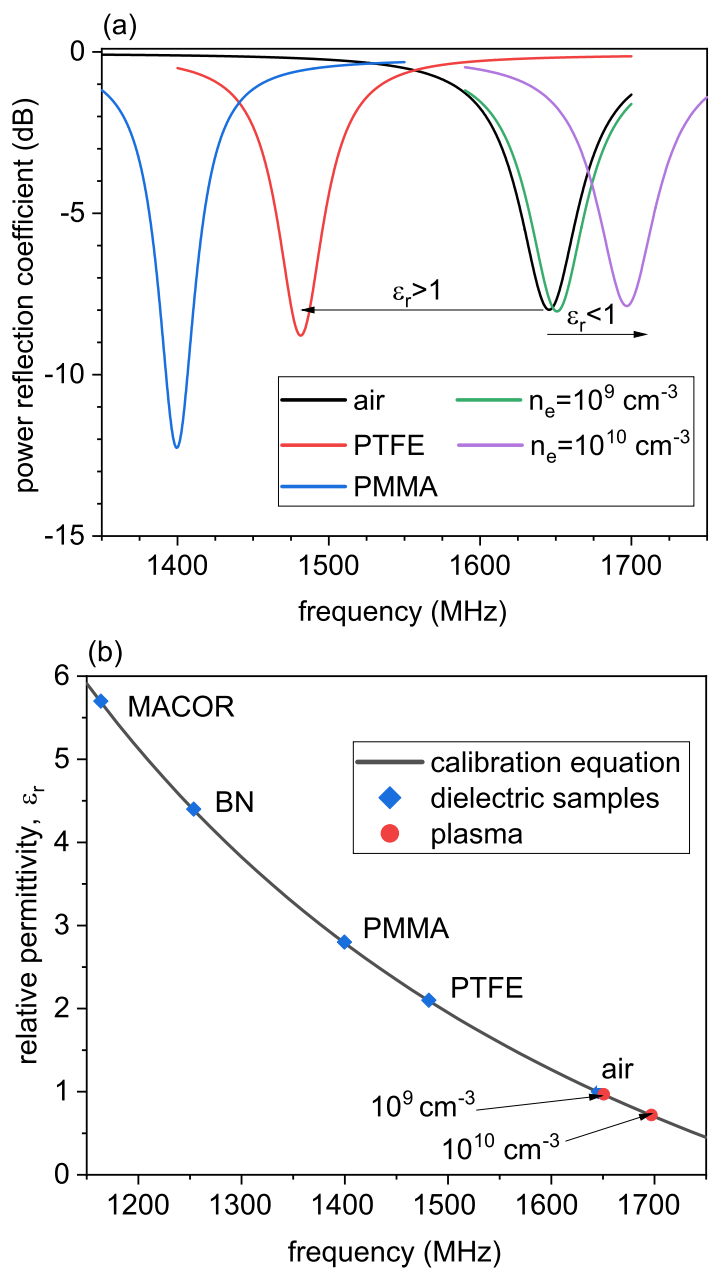

FIG. 6. (a) Simulated resonance spectra for two dielectrics and homogeneous and collisionless plasmas, and (b) the corresponding calibration curve following Equation 6 for the CP1400.

the relative permittivity in the lossy-dielectric range $\left(\varepsilon_{d}>1\right)$ as well as in the plasma domain, $\varepsilon_{p}<1$, even though the probe parameters $\alpha$ and $\beta$ are calculated from solid dielectric material data only. This proves that an absolute calibration procedure can be built by measuring the response of the probe with solid dielectric samples of known permittivity value. Experimental results can then be analyzed without the need for numerous time-consuming numerical simulations. Moreover, 3D numerical simulations are prone to error if the exact geometry of the probe and material properties are not perfectly known.

Behavior in presence of plasma. In the plasma domain, Equation 6 can be combined together with the plasma permittivity resulting in the following relation, similar to the one proposed by Liang et al. [15]:

$$
n_{e}=\frac{4 \pi^{2} m_{e} \varepsilon_{0}}{e^{2}}(1+\alpha)\left(f_{R}^{2}-f_{0}^{2}\right) \quad\left(\mathrm{m}^{-3}\right)
$$

The resonant frequency in presence of cold and collisionless plasma was calculated using 3D simulations for numerous electron densities, ranging from $10^{8}$ to $10^{11} \mathrm{~cm}^{-3}$. Figure 7

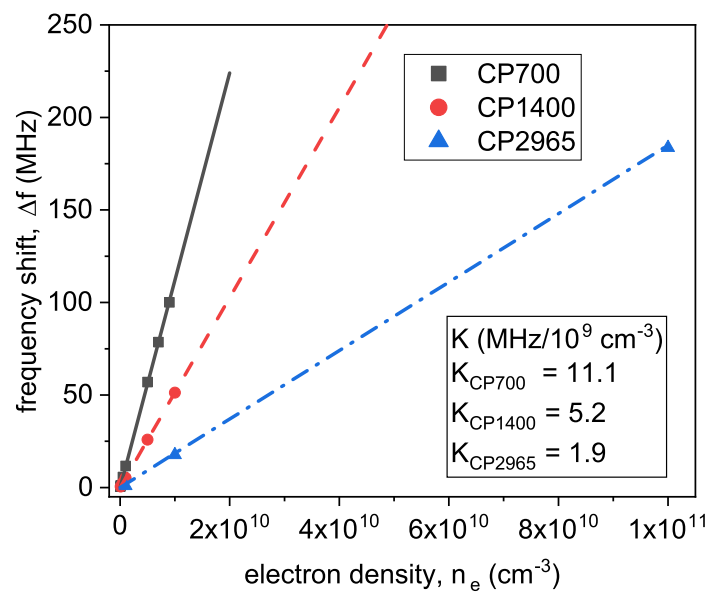

FIG. 7. Simulated sensitivity of the presented probes.

compares the computed frequency shift $v s$ the electron density for the three probes. The probes response appears to be linear for this range of density, and the slope decreases with the vacuum reference frequency (i.e. the working frequency). The best CP sensitivity is obtained for the lowest natural frequency. This means that, for a given electron density, the frequency shift induced by a CP decreases when increasing the vacuum reference frequency. The probe sensitivity is defined in Equation 8, and it is further discussed in section VA

$$
K=\frac{\Delta f}{n_{e}} \quad\left(\mathrm{MHz} / 10^{9} \mathrm{~cm}^{-3}\right)
$$

The importance of this aspect arises when the diagnostic tool is applied to low-density plasmas. For example, if the plasma density to be measured is $10^{8} \mathrm{~cm}^{-3}$, the expected frequency shift $\Delta f$ would be: $1.1 \mathrm{MHz}$ for the CP700, $0.5 \mathrm{MHz}$ for the CP1400 and $0.2 \mathrm{MHz}$ for the CP2965. Letting the precision on frequency detection of the measurement setup be a few hundreds of $\mathrm{kHz}$, it would be easier to get valid and exploitable results using the CP700 rather than the CP2965.

\section{PROBE DESIGN AND EXPERIMENTAL STUDY}

\section{A. Methods and apparatus}

The three manufactured probes CP700, CP1400 and CP2965 are based on a PCB (printed circuit board) spiral resonator: it consists in a $35 \mu \mathrm{m}$-thick copper layer on a dielectric substrate. Two types of dielectric substrate have been used: one is a $1.6 \mathrm{~mm}$-thick FR4 layer, the other is a $0.5 \mathrm{~mm}$ thick RO4003 sheet [26]. FR4 stands for "Flame Retardant $4 \mathrm{n}$ and is a composite material made of woven fiberglass cloth with an epoxy resin binder. The influence of the substrate on the probe is detailed in section IIIC The PCB is mounted on a coaxial SMA connector, with the central pin of the connector being the monopole antenna of the exciting aperture (Figure 1 (a)). Figure 1 (b) shows an example of manufactured probe: CP1400 on a RO4003 dielectric substrate. The diameter of 
TABLE II. Solid dielectrics used for the calibration of the probes.

\begin{tabular}{cccc}
\hline \hline material & $\varepsilon_{d}$ & $\tan \delta$ & reference \\
\hline PTFE & 2.1 & $5 \times 10^{-3}$ & Lide [27] and Beker-Jarvis et al. [28] \\
PMMA & 2.7 & $1.5 \times 10^{-2}$ & Beker-Jarvis et al. [28] \\
BN & 4.4 & $\left(2 \times 10^{-3}\right)$ & Levinshtein et al. [29] (extrapolation) \\
MACOR & 5.7 & $10^{-2}$ & Afsar and Button [30] \\
\hline \hline
\end{tabular}

the probe is $10 \mathrm{~mm}$, which enables a limited but possible spatial resolution. The resonant spectrum of the probe is obtained by measuring the scattering parameter $\mathrm{S} 11$ (power reflection coefficient) with a vector network analyzer (VNA - R\& $S^{\odot}$ ZNB20) used in swept frequency mode. For all measurements presented in this paper, VNA intermediate frequency (IF) filter bandwidth is set to $10 \mathrm{kHz}$, and the frequency resolution is $100 \mathrm{kHz}$. The transmission line (cables and feed-through) is corrected for absorption and reflection using a standard OSM (Open-Short-Match) calibration procedure on the VNA.

\section{B. Probe calibration}

The calibration method presented in section IIB is applied to the probes: resonant spectra are recorded while a solid dielectric sample (with a known permittivity, see Table III) is placed in contact with the probe surface. In Figure 8, the measured spectra used for the calibration of the CP1400 and the derived calibration curve (Equation 6) are presented: experimental data show good agreement with numerical simulations. In the solid dielectric domain Equation 6 returns the relative permittivity value within a margin of error of $\pm 2 \%$. The calibration method being fast and straightforward, each probe was calibrated before the measurement tests described in section IV. The solid dielectric samples have a thickness of at least $10 \mathrm{~mm}$. This necessity arises from the fact that the characteristic decaying length of the emitted EM field is a few $\mathrm{mm}$ [20].

\section{Temperature dependency}

It is noteworthy that the probe temperature can have a significant influence on the reference resonant frequency. This is especially the case for PCB based on classical FR4 substrate. This effect arises from the temperature response of the dielectric substrate: we measured a $4 \%$ increase in FR4 permittivity at $110^{\circ} \mathrm{C}$, yielding a $2 \%$ decrease in the resonant response (since $f_{R} \propto 1 / \sqrt{\varepsilon_{d}}$ ). Though small, this variation corresponds to a frequency drop of approximately $30 \mathrm{MHz}$ for the CP1400. The probe is heated by the surrounding plasma in which the probe is immersed. Temperatures as high as $120^{\circ} \mathrm{C}$ were measured, depending on thruster operating conditions (power and flowrate) and on probe proximity to the thruster.

Correction of the measured resonant frequency. It is important to point out that the dielectric calibration process is done at ambient temperature and that a change in the response of
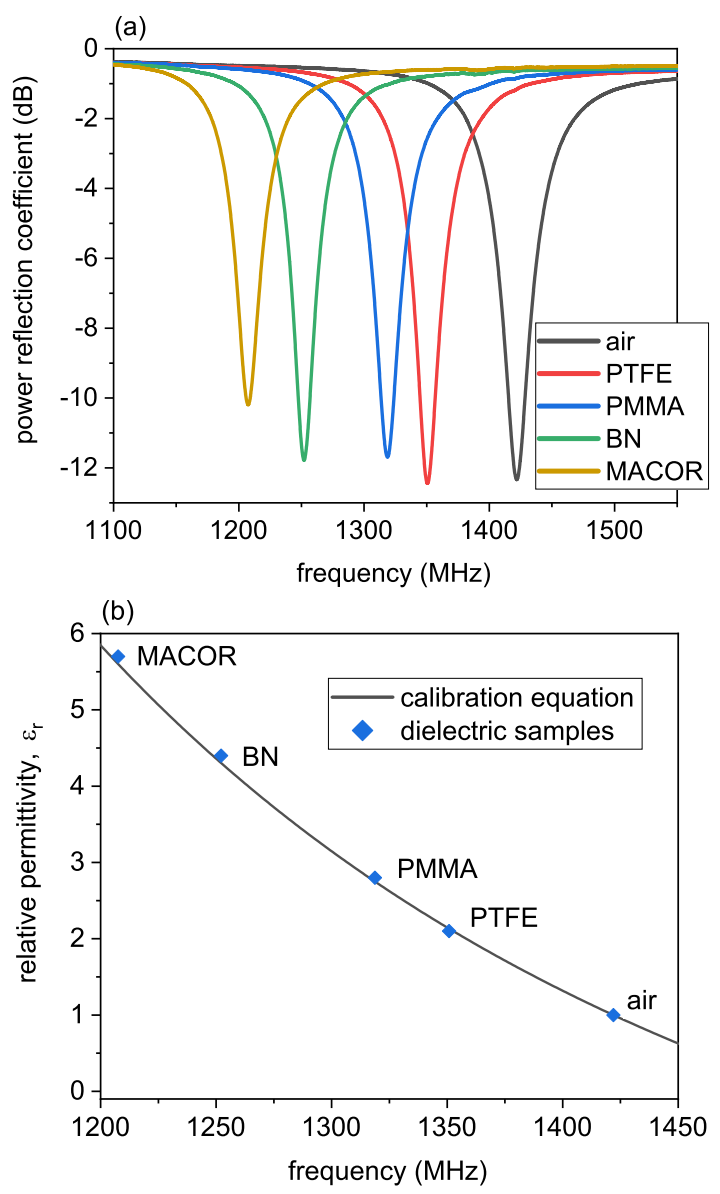

FIG. 8. (a) Measured resonance spectra for the solid dielectrics samples and (b) the corresponding calibration curve following Equation 6 (CP1400).

the probe makes the calibration inapplicable. Hence, it is necessary to precisely know both the temperature and the resonant frequency of the probe when a measurement is performed in the plasma. For this purpose a small $10 \mathrm{k} \Omega$-thermistor is embedded in the FR4-substrate. The vacuum reference frequency is found to vary almost linearly with the temperature, as seen in Figure 9. It is noteworthy that no significant changes in these coefficients have been observed after exposure to plasma.

The frequency shift due to the temperature is corrected through Equation 9.

$$
f_{m}\left(T_{a}\right)=f_{m}\left(T_{p}\right)+\left(f_{0}\left(T_{a}\right)-F\left(T_{p}\right)\right)
$$

where $F(T)$ is the linear fit from Figure 9 and $f_{m}\left(T_{a}\right)$ is the corresponding measured frequency at ambient temperature. Then, the electron density number can be estimated from Equation 7 using $f_{R}=f_{m}\left(T_{a}\right)$. The temperature correction relies on the hypothesis that the frequency shift $(\Delta f)$ between the measured resonant frequency $\left(f_{m}\right)$ and the vacuum reference frequency $\left(f_{0}\right)$ is constant with temperature (Equation 10. The hypothesis has been experimentally validated for temperatures up to $44^{\circ} \mathrm{C}$ with different solid dielectric sam- 


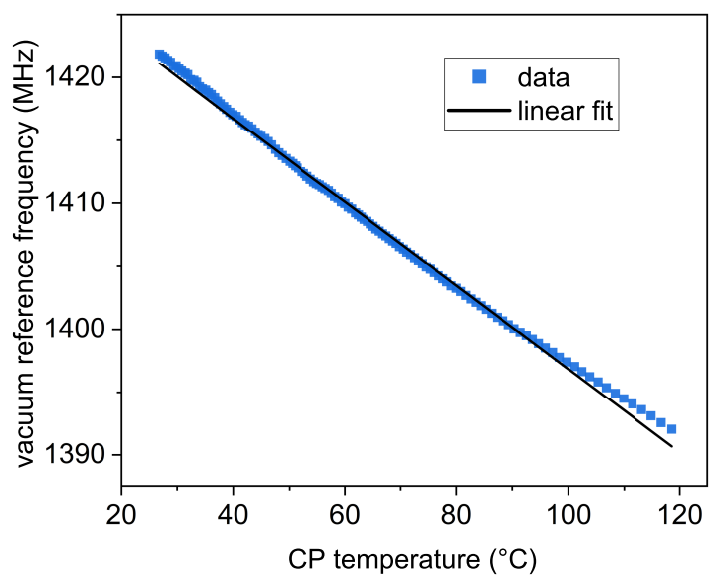

FIG. 9. Variation of the vacuum reference frequency $\left(f_{0}\right)$ vs CP1400 T-Type temperature.

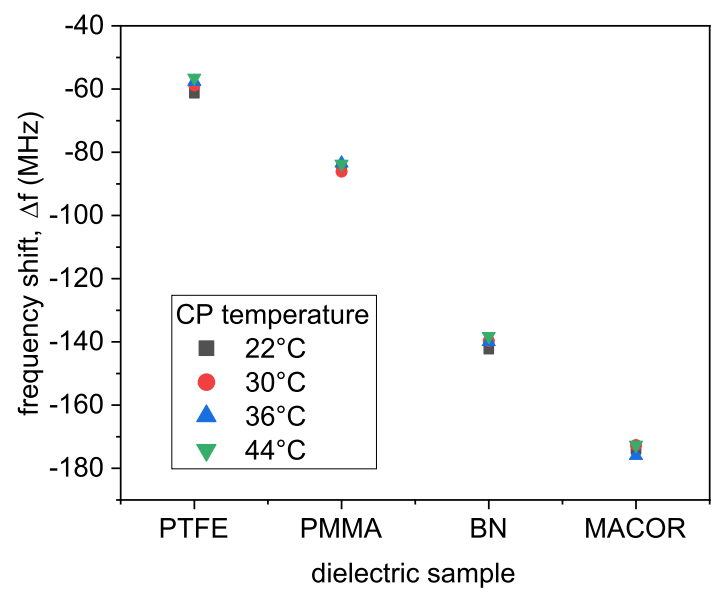

FIG. 10. Measured frequency shift $\Delta f=f_{m}\left(T_{p}\right)-f_{0}\left(T_{p}\right)$ of the available dielectric samples for different probe temperatures (CP1400 T-Type).

ples, as can be seen in Figure 10.

$$
\Delta f=f_{m}\left(T_{p}\right)-f_{0}\left(T_{p}\right)=f_{m}\left(T_{a}\right)-f_{0}\left(T_{a}\right)
$$

Another possible solution is to etch the antenna-slot resonator on a dielectric substrate having a permittivity that is less affected by temperature. This is the case of the RO4003 probes. The temperature coefficient of the dielectric constant of the RO4003 is among the lowest of any circuit board material, and the dielectric constant is stable over a broad frequency range [26]. In this paper, we refer to the FR4-etched probes as "T-Type", and to the RO4003-etched probes as "O-Type".

\section{Measurement uncertainty}

We present here an estimation of the measurement uncertainty for the probes presented in this paper. Five sources of error are considered and summarized in Table III.
- VNA accuracy $\left(\sigma_{V N A}\right)$ is estimated to be less than 10 $\mathrm{KHz}$ in the microwave frequency range of the probes.

- The uncertainty on the measured resonant frequency $\left(\sigma_{f}\right)$, which concerns the vacuum reference and the measurements in presence of plasma, is affected by the spectrum quality. $\sigma_{f}$ has been evaluated from the standard deviation of the measured vacuum reference frequency over 500 measurements performed in representative conditions: the probe is mounted in the vacuum chamber using the same transmission line of plasma measurements and the pressure is in the range $10^{-6}$. $10^{-7}$ mbar.

- The error due to the calibration method $\left(\sigma_{\alpha}\right)$ is the incertitude of the $\alpha$-coefficient in Equation 7. In the plasma domain, 3D numerical simulations show that the deviation between the electron density inferred from the calibration curve and the theoretical density is well below $1 \%$ for $n_{e}$ higher than $10^{9} \mathrm{~cm}^{-3}$. In order to be consistent with the experimental calibration, the uncertainty is taken at $2 \%$, which is the mean deviation observed in the solid dielectric range.

- The error on the corrected experimental frequency due to the temperature correction $\left(\sigma_{T}\right)$ is the mean variance given by the least squares method that returns the linear fit in Figure 9.

- The uncertainty on the vacuum reference frequency $\sigma_{f_{0}}$ results from observed variations of the probe natural frequency that are not corrected (sputtering of the resonator, heating of cables). The uncertainty depends in particular on the exposure length to the plasma. An approximate value of $200 \mathrm{kHz}$ seems to be a good upper limit for these variations.

It should be noticed that the dielectric substrate affects probe performances. Figure 11 (a) shows the normalized reference reflectance spectra -with respect to the spectrum minimum in $\mathrm{dB}$ - for the four manufactured probes and the hairpin $\mathrm{HPw} 44$ (described in section IV A). Table IV summarizes probes performances, and the associated $\sigma_{f}$ and $\sigma_{T}$ uncertainties.

The electron density relative uncertainty $\left(\sigma_{n} / n\right)$ is evaluated following Equation 11

$$
\begin{aligned}
\frac{\sigma_{n}}{n} & =\sqrt{\left(\frac{\sigma_{K}}{K}\right)^{2}+\left(\frac{\sigma_{\Delta f_{m}}}{\Delta f_{m}}\right)^{2}} \\
\frac{\sigma_{K}}{K} & =\sqrt{\left(\frac{\sigma_{\alpha}}{\alpha}\right)^{2}+\left(\frac{\sigma_{f_{0}}}{f_{0}}\right)^{2}} \\
\sigma_{\Delta f_{m}} & =\sqrt{\sigma_{f_{m}}^{2}+\sigma_{f_{0}}^{2}}
\end{aligned}
$$

where $\sigma_{f_{m}}=\sigma_{V N A}+\sigma_{f}+\sigma_{T}$.

The error budget for the different probes is shown in Figure 11 (b). The T-Type probes (etched on a thicker FR4substrate) have larger spectra than the O-Types, which results in a lower quality factor. As can be seen in Figure 11(b), in the range of frequency shift $1 \mathrm{MHz}-100 \mathrm{MHz}$, the CP700 and 
TABLE III. Summary of the considered uncertainties.

\begin{tabular}{lccc}
\hline \hline type & origin & notation & \multicolumn{2}{c}{ value } \\
\hline systematic & VNA accuracy & $\sigma_{V N A}$ & $10^{-1} \mathrm{MHz}$ \\
statistical & uncertainty on the measured resonant frequency & $\sigma_{f}$ & $2 \times 10^{-3}-10^{-1} \mathrm{MHz}$ \\
statistical & error on the relative permittivity calculated with the calibration equation & $\sigma_{\alpha}$ & $2 \%($ relative) \\
statistical & error on the measured resonant frequency due to the temperature correction & $\sigma_{T}$ & $0.02 \%($ relative) \\
statistical & uncertainty on the vacuum reference frequency & $\sigma_{f_{0}}$ & $2 \times 10^{-1} \mathrm{MHz}$ \\
\hline \hline
\end{tabular}

TABLE IV. Estimation of error terms of the presented probes.

\begin{tabular}{ccccccc}
\hline \hline probe & type & $\begin{array}{c}f_{0} \\
(\mathrm{MHz})\end{array}$ & $\begin{array}{c}\mathrm{BW} 3 \mathrm{~dB} \\
(\mathrm{MHz})\end{array}$ & Q factor & $\begin{array}{c}\sigma_{f} \\
(\mathrm{MHz})\end{array}$ & $\begin{array}{c}\sigma_{T} \\
(\mathrm{MHz})\end{array}$ \\
\hline HPw44 & N.A. & 5000 & 100 & 50 & 0.1 & - \\
CP700 & O-Type & 780 & 3.5 & 200 & 0.002 & - \\
CP1400 & O-Type & 1800 & 2 & 900 & 0.02 & - \\
CP1400 & T-Type & 1400 & 15 & 100 & 0.04 & 0.28 \\
CP2965 & T-Type & 3000 & 56 & 60 & 0.06 & 0.6 \\
\hline \hline
\end{tabular}

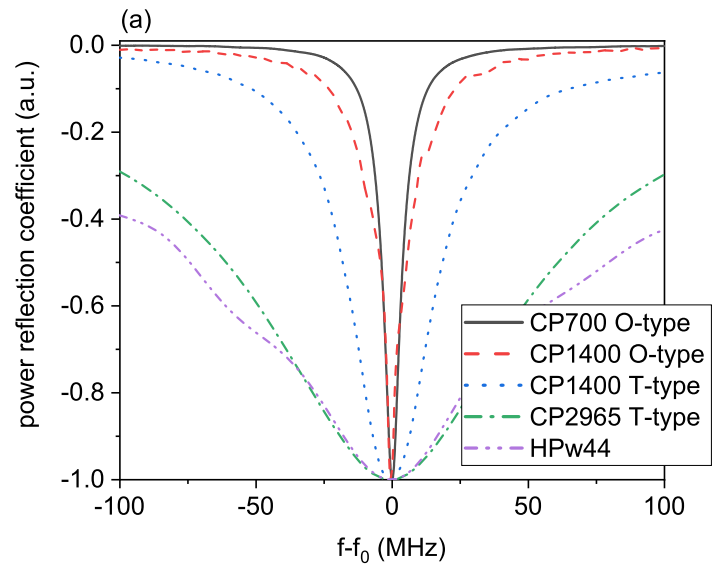

(b)

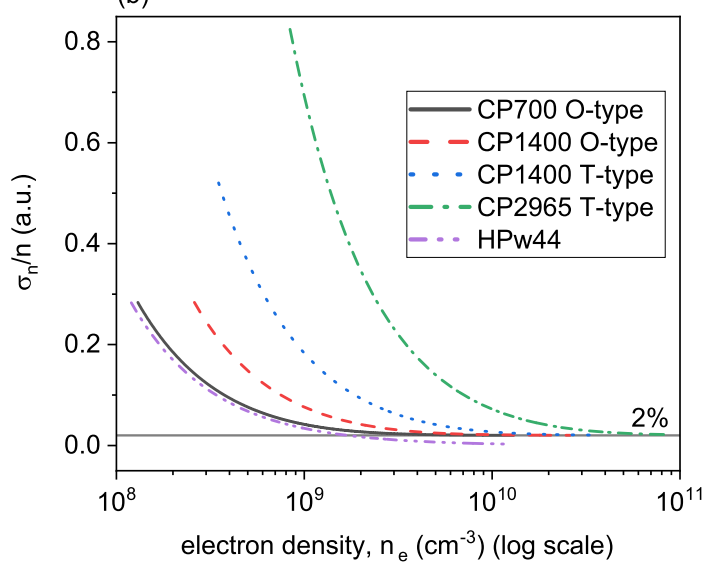

FIG. 11. (a) Non-dimensional reference reflectance spectra of the manufactured probes (CPs and the HP); (b) relative uncertainty $\sigma_{n} / n$ plotted against the electron density $n_{e} . \Delta f$ is in the range $1 \mathrm{MHz}-100$ MHz. the hairpin HPw44 give an estimation of the electron density with an error inferior to $20 \%$ for almost all the range, and the the error on $n_{e}$ is less than $5 \%$ for densities higher than $6 \times 10^{8}$ $\mathrm{cm}^{-3}$. On the other hand, the $20 \%$ precision is only attained for larger densities with the other probes: $4 \times 10^{8} \mathrm{~cm}^{-3}, 10^{9}$ $\mathrm{cm}^{-3}$ and $4 \times 10^{9} \mathrm{~cm}^{-3}$ for the CP1400 O-Type, CP1400 TType and CP2965 T-Type, respectively. It is noteworthy that, for low electron density, the uncertainty is mainly due to the variations of the natural resonant frequency and to temperature corrections, while the error budget is dominated by the error on the calibration method for higher densities. Therefore the relative uncertainty on $n_{e}$ tends to an asymptotic value of $2 \%$ for all curling probes.

\section{APPLICATION TO THE MEASUREMENT OF PLASMA DENSITY ON AN ECR PLASMA THRUSTER}

\section{A. Experimental setup}

The CP performances have been assessed by performing measurements on an Electron Cyclotron Resonance (ECR) plasma thruster. The ECR plasma thruster, which has been developed at ONERA since 2010, is an electrodeless thruster that is based on the resonant absorption of microwaves by electrons to produce the plasma and on the acceleration of electrons and ions in a magnetic nozzle. The originality of this ECR thruster lies in its coaxial geometry that allows a reduction in its diameter and an increase of its performances compared to conventional waveguide ECR sources [31]. Thruster efficiencies between $10 \%$ and $15 \%$ have been obtained with direct thrust measurements. More details about the ECR plasma thruster can be found in [32-35]. To date, few studies on electron density measurements in the plume and the source of electrodeless thrusters have been reported. The electron density has only been measured in the far field region of the ECR plume using LP technique [36]. Theoretical models and numerical simulations of the electron dynamics in the plume of the ECR thruster can be found in Correyero et al. [36], who used a kinetic approach, and Porto and Elias [37], who performed a 1D full-PIC simulation of both the source and the magnetic nozzle. They found the plasma plume to be quasineutral and densities ranging from $10^{11} \mathrm{~cm}^{-3}$, close to the thruster exit section, to $10^{9} \mathrm{~cm}^{-3}$ in the far field region.

The thruster version that is used in this study consists of a $20 \mathrm{~mm}$ long and $27.5 \mathrm{~mm}$ in diameter semi-open coaxial structure, with a back plate made of Boron Nitride. The magnetic field is provided by an annular permanent magnet placed 


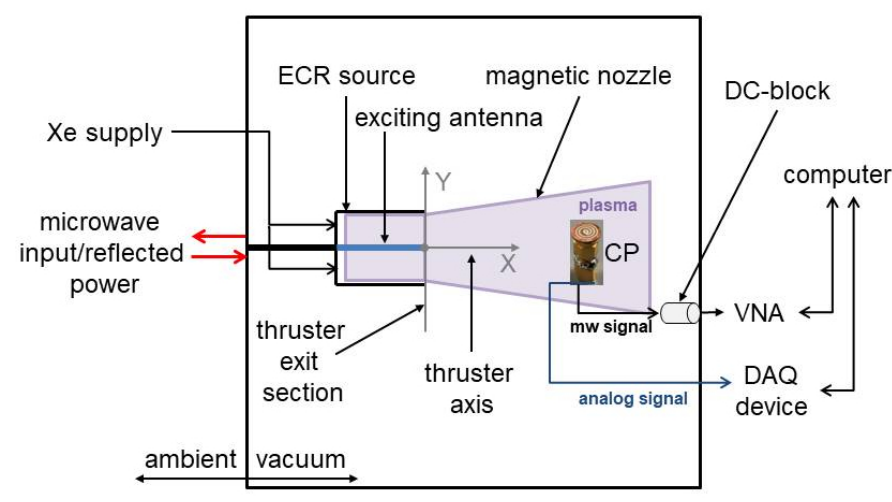

FIG. 12. Schematic of the measurement setup.

at the back of the ECR source. The thruster is powered with a microwave generator at a frequency of $2.45 \mathrm{GHz}$. The power absorbed by the plasma was varied between 15 and $45 \mathrm{~W}$. The thruster is fed with Xenon at a flowrate between 0.8 and 2 $\mathrm{sccm}$. Tests have been carried out inside the ONERA B09vacuum chamber, which is a cylindrical vessel $2 \mathrm{~m}$ long and $0.8 \mathrm{~m}$ in diameter. The secondary pumping system consists of three turbomolecular pumps and one cryogenic pump, yielding a total pumping speed of 13,000 1/s for Xenon, with a base pressure of $10^{-7}$ mbar.

Two types of measurements have been performed: spatial distribution of electron density in the magnetic nozzle plume, where the probe is moved using motorized translation stages, and electron density measurements inside the plasma source, where the probe is flush-mounted in the walls of the ECR source. CP700 and CP1400 have been tested along with a hairpin probe and a Langmuir probe. A home-made hairpin probe, called HPw44, is used for electron density measurements in the plume. It consists of a U-shaped copper antenna of $12.5 \mathrm{~mm}$ in length and with a wire spacing of $\mathrm{w}=4.4 \mathrm{~mm}$, yielding a vacuum reference frequency of $4850 \mathrm{MHz}$. The Langmuir probe consists of a tungsten wire of $0.1 \mathrm{~mm}$ in diameter and $2 \mathrm{~mm}$ in length. A varying bias voltage from $-60 \mathrm{~V}$ to $+100 \mathrm{~V} /+200 \mathrm{~V}$ (according to thruster operating point) was applied at the probe tip and the collected current measured through a $10 \mathrm{k} \Omega$-shunt resistance.

The experimental setup for the measurement of plasma density in the plume is shown in Figure 12. The signals of the thermistor embedded in the "T-Type" CP and of the Langmuir probe are recorded with a National Instrument acquisition card (DAQ device NI BNC-2110). The resonant probes (CPs and HP) are connected to a 4-port VNA, and are left floating at their own potential by insertion of a DC-block in the transmission line, so that no current is drawn from the plasma. The VNA and the DAQ device are connected and remotely controlled by a computer, allowing a good synchronization between the measurements of the CPs temperature and the collection of their resonant spectrum.

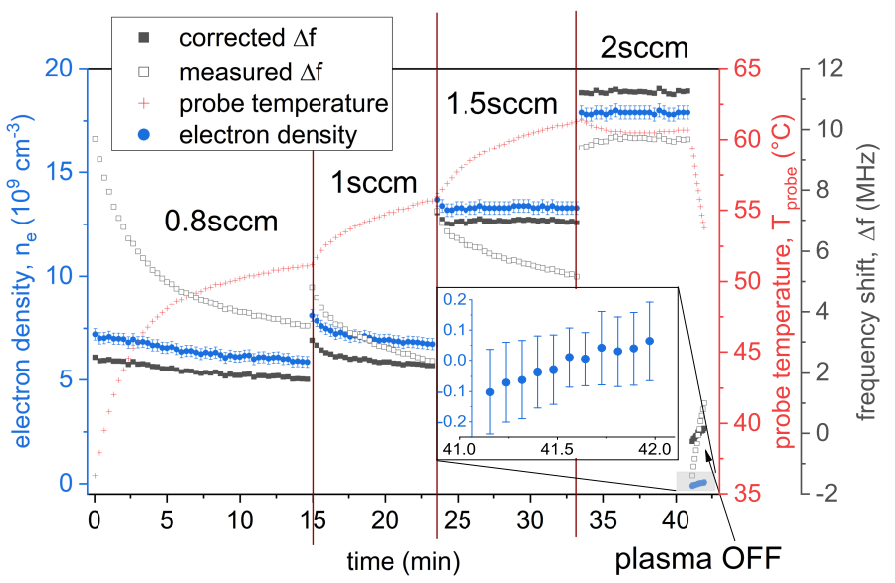

FIG. 13. Electron density measured with CP1400 T-Type at different Xe flowrates and $\mathrm{P}_{\mathrm{abs}}=26 \mathrm{~W}(\mathrm{X}=30 \mathrm{~mm}, \mathrm{Y}=-16 \mathrm{~mm})$. The error bars correspond to the $\sigma$-uncertainty detailed in section IIID

\section{B. Density measurement at a fixed position}

This section presents the density measurements in the thruster plume over a few tens of minutes time span, showing the stability of the diagnostic method together with the effectiveness of the temperature correction for the "T-Type" CPs. Probes position is given with respect to thruster exit section and thruster axis ( $\mathrm{X}$ and $\mathrm{Y}$ axis of Figure 12, with $\mathrm{X}=0$ and $\mathrm{Y}=0$ corresponding to the thruster exit plane and the plume axis, respectively). The thruster is operated with absorbed microwave power $\left(\mathrm{P}_{\mathrm{abs}}\right)$ between 16 and $42 \mathrm{~W}$ and Xenon flowrates between 0.8 and $2 \mathrm{sccm}$. The results are shown in Figures 13 and in 14 for the CP700 and CP1400.

The measured frequency shift $\Delta f$ of the T-Type CP (hollow squares) is strongly affected by the probe temperature and a significant frequency drift occurs during the probe thermal transient (Figure 13). The temperature correction proposed in section III C is applied to the measured $\Delta f$ : the thermal drift is properly corrected over all the acquisition (even during the first phase at $0.8 \mathrm{sccm}$ where a strong temperature gradient is encountered), and the electron density (filled circles) goes back to zero at thruster extinction, with a negligible drift that is in line with the measurement uncertainty (see section III D for numerical values). The measurement is stable in time and the $\mathrm{CP}$ is seen to be sensitive to small variations of electron density.

Figure 14 shows the density measurement performed with the CP1400 T-Type corrected for the probe temperature and CP700 O-Type for various thruster operation set-points. Overall, the density increases with the absorbed power and the flowrate. Electron densities from $8 \times 10^{8} \mathrm{~cm}^{-3}$ to $9 \times 10^{9} \mathrm{~cm}^{-3}$ have been measured. It can be seen that HP and CP results are in good agreement. The CP700 allows more sensitive measurements far from the thruster exit section and is capable of detecting lower densities. 


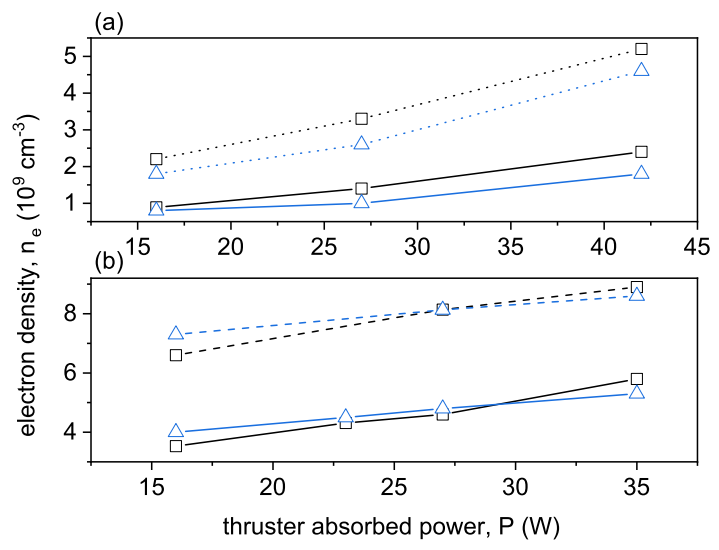

FIG. 14. Measured density for different thruster operating points. (a) CP700 and HPw44 (X=100, Y= $=27 \mathrm{~mm}),($ b) CP1400 and HPw44 $(\mathrm{X}=30, \mathrm{Y}= \pm 21 \mathrm{~mm})$. Squares: CPs, triangle: HPw44. Solid line: 1 sccm, dashed line: $1.5 \mathrm{sccm}$, dotted line: $2 \mathrm{sccm}$.

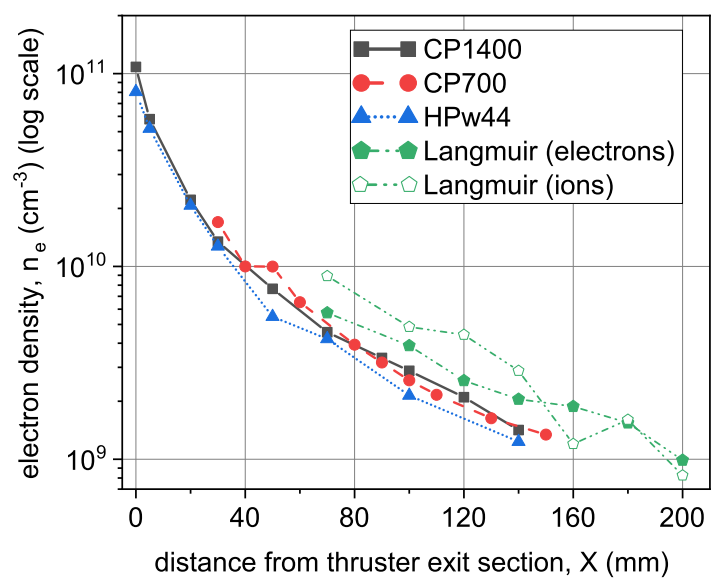

FIG. 15. Longitudinal electron density profiles along the thruster axis at $2 \mathrm{sccm}$ and $\mathrm{P}_{\mathrm{abs}}=27 \mathrm{~W}$.

\section{Spatial distribution of electron density in the plume}

In this section we present the results of the measurements performed in the plume of the ECR thruster along the thruster axis. CP700 and CP1400 O-Type are compared with HP and LP measurements. Figure 15 show the longitudinal electron density profile along the thruster axis measured with the different probes in the same conditions ( $2 \mathrm{sccm}, 27 \mathrm{~W})$. LP measurements can only be performed in the far field region $(X>70$ $\mathrm{mm}$ ) because the probe becomes incandescent when exposed to the plasma flow close to the thruster exit. Nonetheless, LP measurements confirm the hypothesis of quasi-neutral plasma [37] in this region of the magnetic nozzle. Resonant probes are in good agreement with LP results for $X>70 \mathrm{~mm}$. Electron density is decreased from $10^{11}$ at the thruster exit to $10^{9} \mathrm{~cm}^{-3}$ at $\mathrm{X}=140 \mathrm{~mm}$. The region of measurement of the CP700 is limited to $X>30 \mathrm{~mm}$, because the plasma frequency is close to the natural resonant frequency of the probe (this point is developed in section V A.

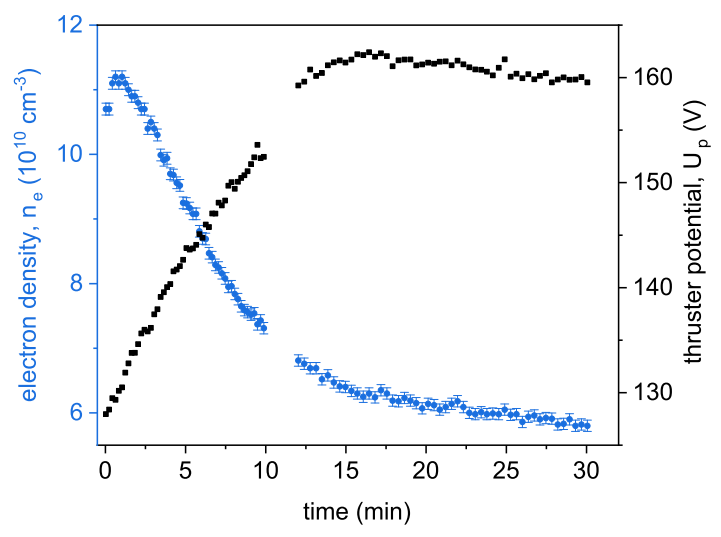

FIG. 16. Time evolution of the electron density inside the source of the ECR thruster (CP2965 T-Type) at $1 \mathrm{sccm}$ and $\mathrm{P}_{\mathrm{abs}}=27 \mathrm{~W}$. The error bars correspond to the $\sigma$-uncertainty detailed in section IIID

\section{Measurement in the ECR source}

The measurement of electron density inside the cylindrical source of the ECR thruster has never been performed before this study as it is a harsh environment for traditional probes (the electron temperature is believed to be of the order of 40 $\mathrm{eV}$, with a plasma density that can reach a few $10^{11} \mathrm{~cm}^{-3}$ ). The CP2965 T-Type is flush-mounted in the cylindrical wall, leaving the probe surface in contact with the plasma. The thruster is operated at $1 \mathrm{sccm}$ and $27 \mathrm{~W}$. The time evolution of the electron density and thruster potential is measured every 10 seconds during the thruster startup, as shown in Figure 16 During the first ten minutes after ignition, a transient is observed, during which the thruster potential $U_{p}$ increases, while electron density decreases. The plasma density in the ECR source is about $6 \times 10^{10} \mathrm{~cm}^{-3}$ after stabilization, which is consistent with values measured during longitudinal scans at $1 \mathrm{sccm}$ in the vicinity of the thruster exit section $\left(n_{e} \geq 5 \times 10^{10}\right.$ $\mathrm{cm}^{-3}$, not shown here). It should be noticed that the ECR source is characterized by the presence of a strong magnetic field (around $500 \mathrm{G}$ at the position of the probe), which is not taken into account by our model (Equation 7).

\section{DISCUSSION}

\section{A. Sensitivity and measurement range of the curling probe}

We discuss now the experimental results presented in section IV starting from the measured probe sensitivity $K$ (defined in Equation 8, , shown in Figure 17. The trend and the orders of magnitude are comparable with the numerical computed values in Figure 7. The linear trend is a first order approximation of Equation 7.

$$
\begin{aligned}
n_{e} & \propto\left(f_{R}^{2}-f_{0}^{2}\right) \\
\left(f_{R}^{2}-f_{0}^{2}\right) & =\left(\Delta f^{2}+2 f_{0} \Delta f\right) \\
n_{e} & \propto 2 f_{0} \Delta f \quad \text { if } \quad \Delta f \ll f_{0}
\end{aligned}
$$




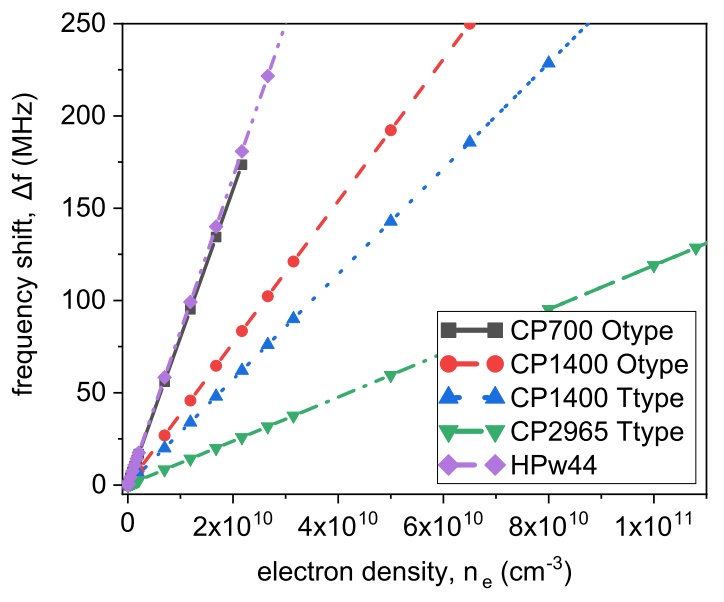

FIG. 17. Measured sensitivity of the tested probes.

TABLE V. $\alpha$-coefficient and probe sensitivity for the presented CPs. O-Type: $0.5 \mathrm{~mm}$-thick RO4003 substrate. T-Type: $1.6 \mathrm{~mm}$-thick FR4 substrate.

\begin{tabular}{cccccc}
\hline \hline probe & type & coating & $\alpha$ & $f_{0}(\mathrm{MHz})$ & $K\left(\mathrm{MHz} / 10^{9} \mathrm{~cm}^{-3}\right)$ \\
\hline CP700 & O-Type bare copper & 6.3 & 780 & 7.1 \\
CP700 & O-Type & BN spray & 9.6 & 730 & 5.2 \\
CP1400 & O-Type bare copper & 4.7 & 1830 & 3.8 \\
CP1400 & O-Type & BN spray & 12.3 & 1730 & 1.75 \\
CP1400 & T-Type bare copper & 11.1 & 1430 & 2.4 \\
CP2965 & T-Type bare copper & 8 & 3010 & 1.5 \\
\hline \hline
\end{tabular}

Hence, the probe sensitivity $K$ is quantified as follows.

$$
\frac{1}{K}=\left\{\begin{array}{l}
\frac{4 \pi^{2} m_{e} \varepsilon_{0}}{e^{2}}(1+\alpha) 2 f_{0} \quad \text { for the CP } \\
\frac{4 \pi^{2} m_{e} \varepsilon_{0}}{e^{2}} 2 f_{0} \quad \text { for the HP }
\end{array}\right.
$$

We remind that, in the case of a $\mathrm{CP}$, the $\alpha$-coefficient is obtained by mean of the calibration procedure section IIIB). The measured probe sensitivities are 7.7, 3.8, 2.9 and 1.2 $\mathrm{MHz} / 10^{9} \mathrm{~cm}^{-3}$ for the CP700 O-Type, CP1400 O-Type, CP1400 T-Type and CP2965 T-Type, respectively. Ergo, for CPs with a similar $\alpha$-coefficient, low-working-frequency probes are better suited for high-accuracy measurements in plasma with a low density. Moreover, the dielectric structure and coating plays an important role: its influence is expressed by the 3D-correction factor $\alpha$-coefficient (see Table V). A thicker dielectric substrate results in a higher $\alpha$-coefficient (and hence in a lower sensitivity). Similarly, the addition of a dielectric coating layer $(\mathrm{BN})$ on the upper side of the resonator significantly increases the value of $\alpha$. The CP700 O-Type has the best sensitivity, close to the employed HP $\left(8.3 \mathrm{MHz} / 10^{9}\right.$ $\mathrm{cm}^{-3}$ ), allowing more precise measurements at lower densities. CP700 sensitivity is consistent with the $\mathrm{CP}\left(f_{0} \approx 870\right.$ $\mathrm{MHz}$ ) used by Hotta et al. [20]: a density of $3 \times 10^{10} \mathrm{~cm}^{-3}$ induces a frequency shift of about $\Delta f=230 \mathrm{MHz}$.

The temperature correction is efficient and it yields stable and consistent measurements when compared to the probes that are not affected by temperature changes, such as the HP
TABLE VI. Frequency shifts and densities for the measured spectra in Figure 18 (a).

\begin{tabular}{ccc}
\hline \hline$\varepsilon_{p}$ & $\Delta f(\mathrm{MHz})$ & $n_{e}\left(\mathrm{~cm}^{-3}\right)$ \\
\hline 0.88 & 7 & $2.7 \times 10^{9}$ \\
0.5 & 33 & $7.6 \times 10^{9}$ \\
0.22 & 56 & $1.1 \times 10^{10}$ \\
0.02 & 80 & $1.4 \times 10^{10}$ \\
-0.5 & 160 & $2 \times 10^{10}$ \\
\hline \hline
\end{tabular}

or the CP O-Type, as can be seen in Figure 14. The temperature drift of the probe recorded signal becomes important when measuring low densities, where a small perturbation on the measured frequency shift could lead to a significant error (that could even result in negative densities if the thermal drift on the frequency shift is larger than the shift produced by the presence of the plasma). The correction effect is observable in Figure 13 at the thruster extinction (plasma OFF): the corrected frequency shift stabilizes over zero while the measured frequency shift significantly drifts.

Hairpin and curling probe measurements are always in good agreement, as can be seen in Figure 14, where the electron density is plotted against the thruster absorbed power. The longitudinal profiles along the thruster axis presented in Figure 15] are consistent with previous LP measurements [38]. The curling probe that is the most adapted to the characterization of the ECR plume in this range of flowrate is the CP1400, as it allows density measurements both in the near field and in the far field plume. The CP700 may be used further away in the plume or at lower flowrates $(<1 \mathrm{sccm})$. The lowest densities that have been measured with the CP700, CP1400 and CP2965 are of the order of $10^{7} \mathrm{~cm}^{-3}, 10^{8} \mathrm{~cm}^{-3}$ and $10^{9} \mathrm{~cm}^{-3}$, respectively. These threshold values result from the sensitivity and the quality factor of the probes.

On the other hand, we observed a limited analyzing range for the CP700. When the plasma density is too high, i.e. when the plasma frequency is larger than the analyzing frequency, the relative plasma permittivity becomes negative $\left(\varepsilon_{p}<0\right)$ and the volume wave takes the form of a decaying surface wave propagating at the interface between the plasma and the probe. This effect can be appreciated observing the measured reflectance spectra from the CP700 for different plasma density (Figure 18 (a)): as the plasma density increases, the resonant frequency shifts to higher values and the reflectance spectrum flattens, meaning that less power is dissipated in the resonator (Table VI). When the critical density value is reached, the surface wave abruptly decays at the plasma-probe interface, and is completely confined inside the structure under the antenna slot, where plasma is not present, resulting in a negative frequency shift $\left(f_{R}<f_{0}\right)$. This effect can be observed on Figure 18 (b): as the plasma density approaches the critical value, the surface wave is constrained at the probe interface, being reflected by the mirroring plasma. That is the case for the blue spectrum that measures a density of $2 \times 10^{9} \mathrm{~cm}^{-3}$, whereas the expected value is $3.3 \times 10^{10} \mathrm{~cm}^{-3}$ (CP1400 OType), yielding a relative plasma permittivity of -2 (if evaluated at the CP700 working frequency). When the plasma den- 


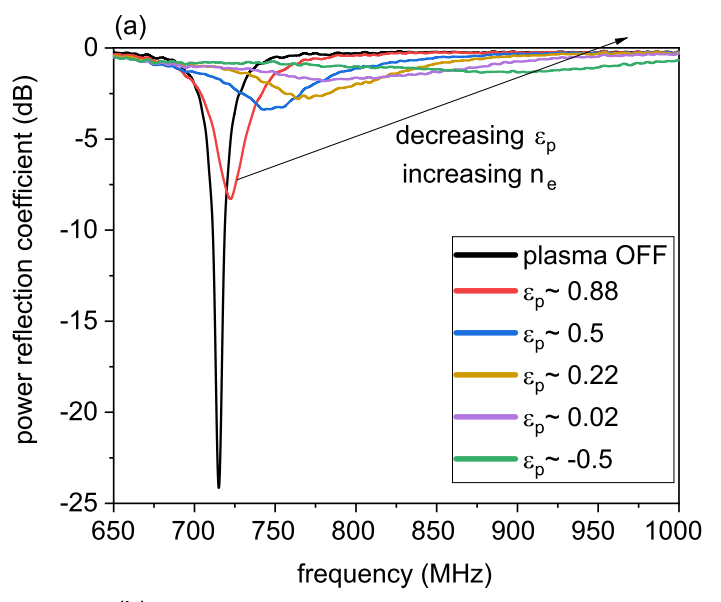

(b)

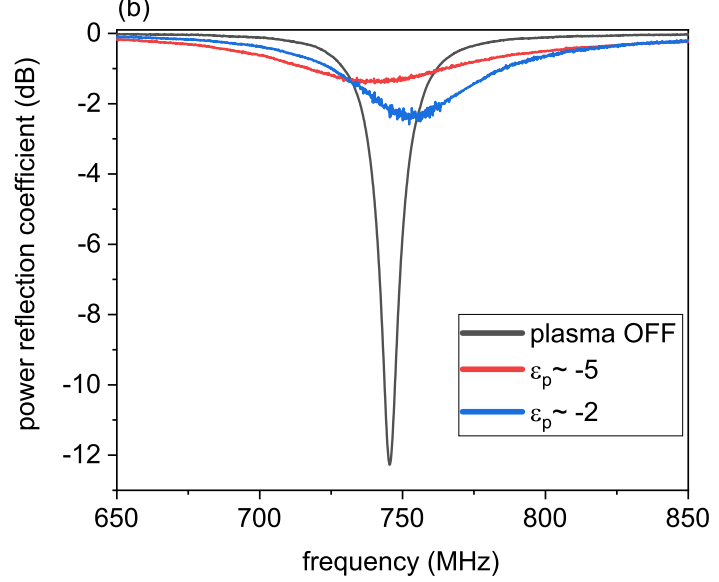

FIG. 18. (a) Measured reflectance spectra when increasing plasma density and (b) the effect of saturation for the CP700.

sity is larger than the critical value $\left(5.8 \times 10^{10} \mathrm{~cm}^{-3}\right.$ measured with the CP1400 O-Type, yielding $\varepsilon_{p}=-5$ ), the wave only exists inside the probe, where the substrate permittivity larger than 1 induces a left-shift of the resonant frequency, resulting in the nonsensical detection of a negative density (red spectrum).

\section{B. The second harmonic resonance}

One way to overcome the issue of limited measurable density range of the CP700 is to use its second harmonic resonance (SHR). The ratio between the SHR and the first harmonic resonance (FHR) is 2.3 due to the spurious resonant nature, i.e. in-between the quarter- and the half-wavelength resonance. The SHR has been simulated and validated by experiments. The SHR behaves as the FHR (Figure 19), so that the calibration procedure (section IIIB can be applied to the SHR as well. Therefore, SHR could be used to perform measurements in conditions where the FHR fails, but with degraded performances : the reflectance spectrum has a reduced amplitude with respect to FHR and has a larger bandwidth, hence a higher uncertainty on the measured frequency. Table VII gives the absolute and relative frequency shift in presence of PTFE
TABLE VII. Frequency shift of FHR and SHR of the CP700.

\begin{tabular}{ccc}
\hline \hline dielectric & FHR $\Delta f\left(\Delta f / f_{0}\right)$ & SHR $\Delta f\left(\Delta f / f_{0}\right)$ \\
\hline PTFE & $-58 \mathrm{MHz}(0.074)$ & $-95 \mathrm{MHz}(0.055)$ \\
PMMA & $-82 \mathrm{MHz}(0.105)$ & $-126 \mathrm{MHz}(0.073)$ \\
\hline \hline
\end{tabular}

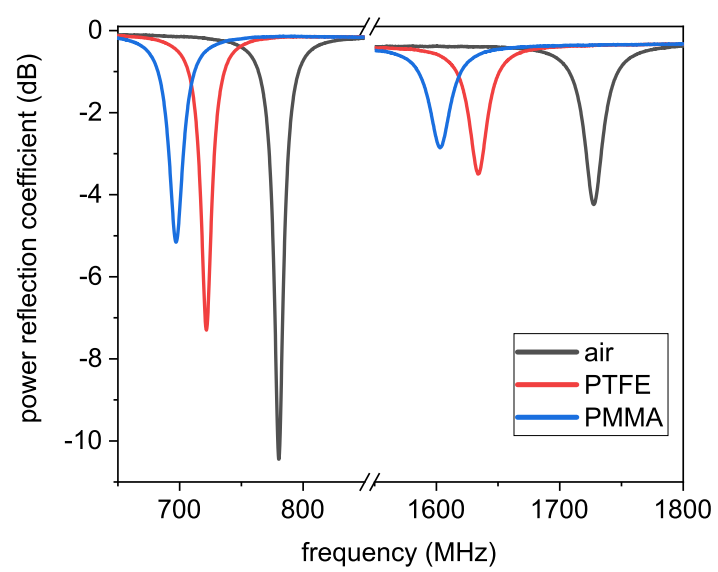

FIG. 19. Measured reflectance spectra in presence of solid dielectrics of the FHR and the SHR for the CP700.

and PMMA (solid dielectrics samples): the SHR relative frequency shift is lower than the FHR. In addition, the calibration coefficient $\alpha$ differs (6.3 and 11.4 for the FHR and the SHR respectively), yielding a plasma sensitivity lower than the FHR $\left(K_{F H R}=7.7 \mathrm{MHz} / 10^{9} \mathrm{~cm}^{-3}\right.$ and $K_{S H R}=1.9 \mathrm{MHz} / 10^{9}$ $\mathrm{cm}^{-3}$ ). Figure 20 shows an example of application during a plasma measurement where FHR could not be used. Electron density obtained from SHR $\left(9 \times 10^{9} \mathrm{~cm}^{-3}\right)$ is in line with measurements performed with CP1400 $\left(10^{10} \mathrm{~cm}^{-3}\right)$. Further investigations on the possibilities of performing plasma measurements using the SHR are ongoing. It is worth mentioning that the use of similar higher resonance modes has been proposed by Liang et al. [39] for a hairpin resonator.

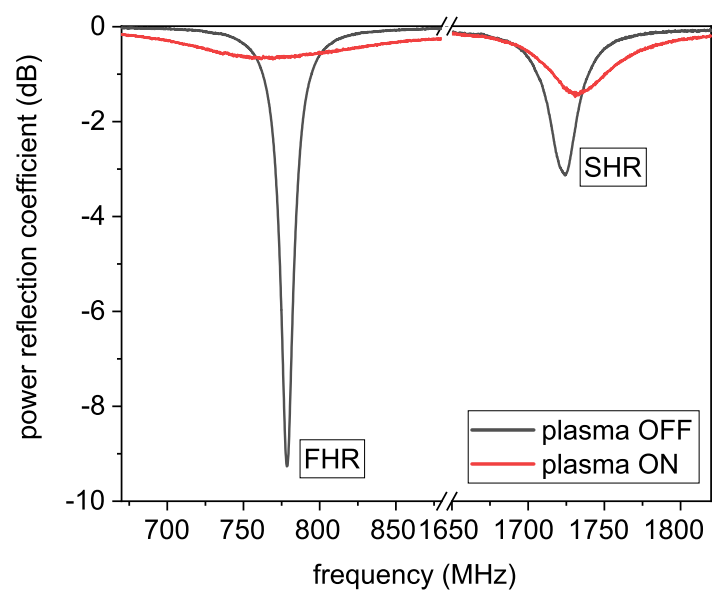

FIG. 20. Measured FHR and SHR reflectance spectra in presence of plasma for the CP700. 


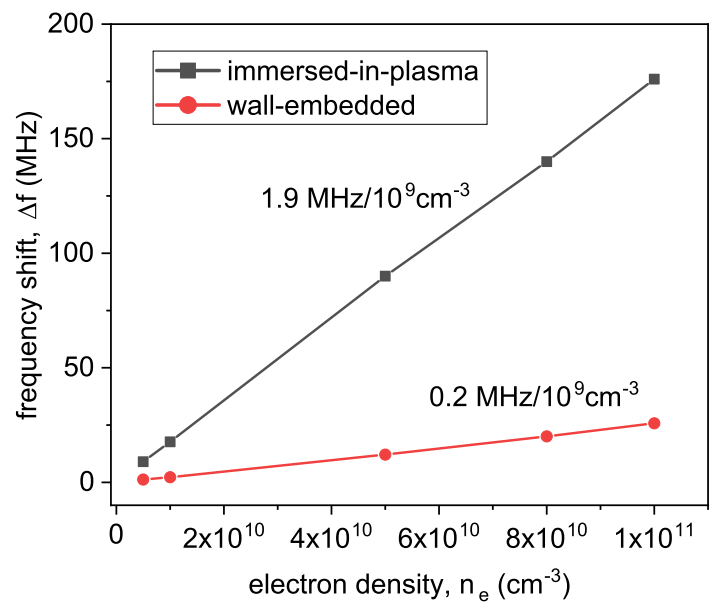

FIG. 21. Simulated immersed-in-plasma $v s$ wall-embedded CP2965 frequency shift for different plasma densities.

\section{Towards a fully-non-intrusive diagnostic method}

CP2965 small-dimensions allowed its insertion inside the plasma source (of outer diameter $41.5 \mathrm{~mm}$ ). The probe being in direct contact with the plasma, it strongly suffered from plasma etching and surface interaction with plasma. To avoid direct contact with the high-density, high-temperature plasma inside the source, the CP could be embedded in the dielectric wall of the thruster source chamber, leaving a dielectric layer of a few mm between the CP surface and the plasma. Thus, a fully non-intrusive diagnostic tool could be obtained, as the $\mathrm{CP}$ surface would not be in contact with the plasma. Figure 21 shows the numerical simulation of the immersed-in-plasma $v s$ wall-embedded frequency shift for different plasma densities for a CP2965 embedded in a $1 \mathrm{~mm}-\mathrm{BN}$ chamber wall. The frequency shift is reduced by roughly 10 times with respect to the immersed-in-plasma probe case; even so, probe response is still exploitable for plasma densities higher than $10^{10} \mathrm{~cm}^{-3}$. In such case, the calibration process should take into account the wall thickness by covering the probe surface with a $1 \mathrm{~mm}$ thick BN layer, prior to the placement of the solid dielectric sample.

\section{CONCLUSION}

In this work, we presented the numerical and experimental study of the curling probe [15]: a spiral slot-type planar resonant probe. The $2 \mathrm{D}$ numerical study led to a better understanding of the spiral resonator, establishing the equation governing the 2D resonant frequency as a function of three non-dimensional geometrical parameters. The $3 \mathrm{D}$ analysis led to the determination of a calibration process, returning the 3D correction factors $(\alpha$ and $\beta$ ) that account for the structure and the coupling effects. This calibration process is based on solid dielectric etalons with known relative permittivities (between 2.1 and 5.7). Numerical simulations show that the resonant frequency shift in the plasma domain is well predicted by the calibration curve, meaning that no reference plasma source is required to calibrate the probe. Moreover, the probe calibration is validated by comparison of electron density measurements with other techniques (hairpin and Langmuir probes). Three CPs at different working frequencies have been designed: CP700, CP1400 and CP2965; the number being approximately the vacuum reference frequency in $\mathrm{MHz}$. Simulations indicate that the low frequency probes have a higher sensitivity while being limited in their electron density measurement range. The CPs have been manufactured by etching process on two different dielectric substrates: FR4 and RO4003. FR4-based CPs are embedded with a thermistor in order to measure their temperature while the probe is exposed to the plasma. This allows to correct the resonance frequency drift due to the variation of permittivity with temperature. The resonance drift on RO4003-based CPs is seen to be negligible.

This paper shows the interest of the microwave-resonant probe technique by means of a curling probe for the measurement of electron density in electric propulsion systems. The CPs have been tested in the plume and in the source of an ECR plasma thruster fed with Xenon. The reliability and the stability over time of the measurement has been proven both in the plume and in the source of the thruster: at low electron densities and background pressures $\left(10^{8}-10^{10} \mathrm{~cm}^{-3}\right.$ and $\left.10^{-6}-10^{-5} \mathrm{mbar}\right)$, and at higher electron densities and background pressures $\left(10^{11} \mathrm{~cm}^{-3}\right.$ and $\left.10^{-3} \mathrm{mbar}\right)$, respectively. Tests showed that low-working frequencies probes (CP700) are better suited for measuring low density plasma but suffer from a low critical density $\left(\sim 10^{10} \mathrm{~cm}^{-3}\right)$. On the opposite, the CP2965 proved to withstand high density and high electron temperature plasma. A totally-non-intrusive diagnostic technique could be obtained by embedding the CP2965 inside the wall of the source.

The analytical relation between the $3 \mathrm{D}$ and the $2 \mathrm{D}$ resonant frequency is currently under investigation as well as the the impact of the plasma sheath that may form at the probes surface. Along with time-resolved electron density measurements and the integrability of a high-frequency CP inside a thruster plasma source, these subjects are part of the oncoming works on the curling probe and its applications to electric propulsion diagnostics.

\section{DATA AVAILABILITY STATEMENT}

The data that support the findings of this study are available from the corresponding author upon reasonable request.
[1] N. Hershkowitz, M. H. Cho, and C. H. Nam, Plasma Chem Plasma Process 8, 35 (1988)
[2] F. F. Chen, J. D. Evans, and W. Zawalski, Plasma Sources Sci. Technol. 21, 055002 (2012) 
[3] F. F. Chen, Introduction to Plasma Physics and Controlled Fusion (Springer, 2018).

[4] T. Shirakawa and H. Sugai, Jpn. J. Appl. Phys. 32, 5129 (1993)

[5] D. R. Bates, A. E. Kingston, and R. W. P. McWhirter, Proc. R. Soc. Lond. A 267,297 (1962).

[6] H. Griem, Principles of Plasma Spectroscopy (Cambridge University Press, 1997).

[7] I. H. Hutchinson, Princ. Plasma Diagnostics (Cambridge University Press, 2002).

[8] M. D. Bowden, Y. Goto, H. Yanaga, P. J. Howarth, K. Uchino, and K. Muraoka, Plasma Sources Sci. Technol. 8, 203 (1999)

[9] E. A. D. Carbone, S. Hubner, J. M. Palomares, and J. J. A. M. van der Mullen, J. Phys. D Appl. Phys. 45, 345203 (2012).

[10] B. Vincent, S. Tsikata, S. Mazouffre, T. Minea, and J. Fils, Plasma Sources Sci. Technol. 27, 055002 (2018)

[11] R. L. Stenzel, Rev. Sci. Instrum. 47, 603 (1976)

[12] R. Piejak, in Gaseous Electron. Conf. (Shannon, Ireland, 2004) p. ES2.011.

[13] R. B. Piejak, V. A. Godyak, R. Garner, B. M. Alexandrovich, and N. Sternberg, J. Appl. Phys. 95, 3785 (2004)

[14] A. Pandey, H. Tashiro, W. Sakakibara, K. Nakamura, and H. Sugai, Plasma Sources Sci. Technol. 25, 065013 (2016)

[15] I. Liang, K. Nakamura, and H. Sugai, Appl. Phys. Express 4, 066101 (2011)

[16] A. Arshadi and R. P. Brinkmann, Plasma Sources Sci. Technol. 25, 45014 (2016)

[17] M. A. Lieberman and A. J. Lichtenberg, Principles of plasma discharges and material processing (Wiley, 2005).

[18] A. Arshadi and R. P. Brinkmann, Plasma Sources Sci. Technol. 26, 015011 (2017).

[19] A. Pandey, W. Sakakibara, H. Matsuoka, K. Nakamura, and H. Sugai, App. Phys. Lett. 104, 024111 (2014)

[20] M. Hotta, D. Ogawa, K. Nakamura, and H. Sugai, Jpn. J. Appl. Phys 57, 046201 (2018)

[21] D. Ogawa, K. Nakamura, and H. Sugai, Plasma Sources Sci. Technol. 29, 075009 (2020)

[22] D. Langmuir, E. Stuhlinger, and J. Sellen, Electrostatic Propulsion (Academic Press, New York, 1961).
[23] R. B. Lobbia and B. E. Beal, J. Propuls. Power 33, 566 (2017)

[24] C. Deline, B. E. Gilchrist, C. Dobson, J. E. Jones, and D. G. Chavers, Rev. Sci. Instrum. 78, 113504 (2007)

[25] A. Arshadi, R. P. Brinkmann, M. Hotta, and K. Nakamura, Plasma Sources Sci. Technol. 26, 045013 (2017)

[26] Rogers Corporation, "RO4000 Series Data Sheet,"

[27] D. R. Lide, CRC Handbook of Chemistry and Physics, 85th ed. (CRC Press/Taylor and Francis, 2005).

[28] J. Beker-Jarvis, M. D. Janezic, B. F. Riddle, R. T. Johnk, P. Kabos, C. L. Holloway, R. G. Geyer, and C. A. Grosvenor, NIST Tech. Note 1536. Tech. Rep. (2005).

[29] M. E. Levinshtein, S. L. Rumyantsev, and M. S. Shur, Properties of Advanced Semiconductor Materials: GaN, AIN, InN, $B N$, SiC, SiGe (Wiley, 2001).

[30] M. N. Afsar and K. Button, IEEE Trans. Microw. Theory Tech. 31, 217 (1983).

[31] S. Peterschmitt, Development of a stable and efficient electron cyclotron resonance thruster with magnetic nozzle, Ph.D. thesis, Institut Polytechnique de Paris (2020).

[32] J. C. Sercel, An experimental and theoretical study of the ECR plasma engine, Ph.D. thesis, California Institute of Technology (1993).

[33] F. Cannat, T. Lafleur, J. Jarrige, P. Chabert, P. Q. Elias, and D. Packan, Phys. Plasmas 22, 053503 (2015)

[34] J. Jarrige, P.-Q. Elias, D. Packan, and F. Cannat, in 44th AIAA Plasmadynamics Lasers Conf. (2013) p. 2628.

[35] T. Vialis, J. Jarrige, A. Aanesland, and D. Packan, J. Propuls. Power 34, 1323 (2018)

[36] S. Correyero, J. Jarrige, D. Packan, and E. Ahedo, in Sp. Propuls. 2018, May (Seville, Spain, 2018).

[37] J. C. Porto and P.-Q. Elias, in 36th Int. Electr. Propuls. Conf., October (Vienna, Austria, 2019) pp. IEPC-2019-232.

[38] S. Correyero, J. Jarrige, D. Packan, and E. Ahedo, Plasma Sources Sci. Technol. 28, 095004 (2019)

[39] Y. Liang, K. Kato, K. Nakamura, and H. Sugai, Jpn. J. Appl. Phys. 50, $116101(2011)$ 